\title{
Solar Modulation on Galactic Cosmic Rays in the Earth's Atmosphere
}

\author{
UMAHI, A.E. \\ Department of Industrial physics (Astrophysics unit), Faculty of Science, Ebonyi State University, Abakaliki, \\ Nigeria.
}

\begin{abstract}
A brief review of solar modulation on galactic cosmic rays in the earth's atmosphere is presented. The results of the characterizations of the major two events i.e. Galactic Cosmic Rays (GCRs)(measured in counts) and Galactic Solar rays(GSRs) (measured in counts) against time (measured in hour) shows significant variations. It was also observed that the variations of the events are slightly out of phase. The anti-correlation coefficient, $\boldsymbol{r}$ between GCRs and GSRs, ranging from -0.015 to -0.470, shows that the events originates from different sources. The low level of $\boldsymbol{r}$ in this result indicates that other solar activities such as sunspot, coronal mass ejection and solar wind directly enters the Earth's atmosphere.
\end{abstract}

Keywords: Cosmic Rays, Solar rays, Modulation and Solar Activity.

\section{Introduction}

The investigation of cosmic ray intensity at various time scales and under the condition of solar magnetic activity is important to the understanding of the dynamics of the earth's atmosphere which is responsible for the modulation of galactic cosmic rays (GCRs) $[1,2,3,4,5,6,7]$.The energy spectra and absolute fluxes of cosmic-ray proton and heliumconstitute the most fundamental data in the study of cosmic-ray physics. Their interstellarspectra carry the information on the origin and propagation history of the cosmicrays in the Galaxy. However, observable spectra in the heliosphere are deformedby the solar modulation effect, and balloon data are also affected by the atmosphericinteractions. In order to obtain the interstellar spectra, the understanding ofthe solar modulation and precise estimation of the atmospheric effects are very important[8].

The quantitative attractions of the large differences in the time lags for a cycle of solar modulation on galactic cosmic rays propagation in the earth's atmosphere is highly debated on simple interpretation and use of sunspot numbers as the appropriate index of solar activity. The galactic cosmic rays flux data observed by ground based stations/networks at the earth's surface are within eleven years cycle. It was found that the cosmic rays intensity decreasing with increasing solar activity [9].

When thegalacticsolar rays and galactic cosmic rays propagates through the heliosphere, they introduce ionization in the process. The increasingrate of ionization that takes place is alarming in the earth's heliosphere and the radiationsarethe most dangerous emission of protons, $\mathrm{x}$ - rays and ultraviolent radiations. Due to these atmospheric contributions, they continuous monitoring of the Earth's upper environment remains an important region for astronomers, astrophysics and space scientist globally [10].

In the past decades, some scientists monitored and measured the induced ionization at different locations and during solar cycles with the early Balloon experiments [11,12,13,14,15,], Rockets[16], Spacecrafts [17], Muon telescope[18] and ground base observatory networks [19]. The ground base observatory networks are introduced in the observationof ionization rates and level in the environment. The locations of some ground base observatories for cosmic rays measurement are as follows: Mirny, Antarctica $\left(66^{\circ} 33^{\prime} \mathrm{S}\right.$; $\left.93^{0} 00^{\prime}\right)$; Tixie $\left(71^{0} 33^{\prime} \mathrm{N} ; 128^{0} 54^{\prime}\right)$; Murmansk Region $\left(68^{0} 59^{\prime} \mathrm{N}\right.$; $\left.33^{\circ} 0 \mathrm{~S}^{\prime}\right)$; Norilsk $\left(69^{\circ} 00^{\prime} \mathrm{N} ; 88^{0} 00^{\prime}\right)$; Moscow Region $\left(55^{0} 28^{\prime} \mathrm{N}\right.$; $\left.37^{0} 19^{\prime}\right)$; Alma-Ata $\left(43^{0} 12^{\prime} \mathrm{N}\right.$; $\left.76^{0} 56^{\prime}\right)$; Erevan $\left(40^{0} 10^{\prime} \mathrm{N} ; 44^{0} 30^{\prime}\right)$, Forschungszentrum Karlsruhe, Germany $\left(49^{\circ} \mathrm{N}, 8^{0} \mathrm{E}\right)$ and Sea Expeditions $\left(60^{\circ} \mathrm{N}, 60^{\circ} \mathrm{S}\right)$. In addition, the National Aeronautics and Space Administration (NASA) is monitoring the space with Bonner Ball Neutron Detector (BBND), Charged Particle Directional Spectrometers (CPDS), Dosimetric Mapping (DOSMAP), Radiation Doses Experienced by Astronauts in EVA (EVARM), Passive Dosimetry (PD), Phantom Torso (PT) and Tissue Equivalent Proportional Counter (TEPC).

Recently, the BESS balloon [20, 21, 22, 23, 24]flights were performed in Northern Canada from Lynn Lake ( 0.4 GV cutoff rigidity), Manitoba to Peace River, Alberta in summer of 1997 to 1999 and 2000. Within these flights, the BESS reached at a float altitude $\mathrm{f} 36 \mathrm{~km}\left(\sim 5 \mathrm{~g} / \mathrm{cm}^{2}\right.$ of residual atmosphere). In the four years, solar activity have changed from minimum to maximum as known from the sunspot numbers and neutron monitor which are available in http://odysseus.uchicago.edu/NeutronMonitor/: University of Chicago, "National Science Foundation Grant ATM-9613963". They also obtained the ascending data of cosmic rays in1999 and 2000 and the descending data at Ft. Sumner (4.2 GVcut off rigidity), New Mexico, USA in 2001 [25].In particular, the BESS-2001 data enables the comparison of secondary proton in low energy region below the 
cutoff. These data improved the parameters of elementary which processes to reproduce the observed data better at various altitudes[26].

The ionization chamber data of solar cycle 17 and 18 showed that the cosmic rays variations lagged behind solar activities, such as measured sunspot number by $6-12$ months [27,28]. The solar cycle 19 with neutron monitor data attributed the variation to the dynamics and inertia of the modulating media [29]. The time lag, $\Delta \mathrm{t}$, of the modulating medium was expressed in detail [30,31,32,33]. The neutron monitoring has a threshold rigidity of $2.2 \mathrm{GV}$ and its response to the nucleonic component of cosmic ray was typical of a high latitude sea-level station[30].

The solar cycle 20 studied the relationship between the cosmic rays intensity and solar activities over the last two solar cycle (1955-1976) using neutron data monitor which was provided through the online database of the Polar Geophysical Institute of the Russian Academy of Sciences (Apatity, geomagnetic cut-off 0.6 GV) or the Sodankyl Geophysical Observatory of the University of Oulu, Finland $(0.8 \mathrm{GV})$ or the Solar-Terrestrial Physics Division of Izmiran, Russia (Moscow, $2.4 \mathrm{GV}$ ) or the Space Physics Data System of the University of New Hampshire, USA (Climax, 3.0 GV and Haleakala, 12.9 GV) or the Irkutsk Neutron monitor, the Unit of Space Physics at North-West University Potchefstroom, South Africa (Potchefstroom: 7.0 GV and Tsumeb, Namibia: 9.2 GV), or the Institute of Space Physics Beijing, China (9.6 GV). The cosmic ray intensity lag sunspot number during solar cycle 19 and up to solar maximum in 1969. However during 1971-1972 the highest cosmic ray intensity was recorded with more gradually decreasing sunspot number[30].A correlation analysis between the monthly cosmic ray intensity and the monthly sunspot number showed that for solar cycle 19, the cross correlation coefficient was a maximum for time lag of $\sim 12$ month which is in agreement with Pomerantz and Duggal (1974) [34]. In the solar cycle 20, the maximum correlation occurred for a much shorter time lag of two months. Thus, during solar cycle 20 , the activity was less than the previous cycle which indicates that the time lag of the solar activity were less. If this time lag was used in the interpretation of the measured size of the heliosphere, then it appears that the dimension of the heliosphere is not constant. But it depends upon the level of activities during a given solar cycle. Therefore, they suggested that the heliosphere was'expanded' to a large size during more activity cycles. In addition, the measurements of low energy cosmic-ray proton and helium spectra by the BESS spectrometer were used to discuss the solar modulation effects usingthe observed data between 1997 to 2000 [35].This work will focus on the solar modulation on galactic cosmic rays in the earth's atmosphere where the source of particles considered the cosmic rays and solar flare. The main procedure of this work is to statistically analysis the hourly variations of cosmic rays and solar flare in the earth's atmosphere using excel programs.

\section{Solar Modulation}

The solar modulation of galactic cosmic rays has been studied for several decades, it is still a subject of intense research to access the continuous changing behaviors of the sun activities' and its influence on cosmic rays. The galactic cosmic rays are subjected to solar variations modulations in the earth's atmosphere. This modulation of cosmic rays intensity are associated with the eleven years solar activity cycle [36, 37, 38]. The influence of solar output modulation was first studied by Forbush (1958) [28] and many subsequent researchers $[39,40,41,42,43,44]$. The solar magnetic field reverses at each solar activity maximum resulting in the 22 year cycle as well $[45,46]$. Therefore, the solar magnetic field is based on the orientationfield which may be either positive or negative.The positive orientationfield defineswhen the field outward from the sun is in the Northern heliosphere (e.g during the 1970's and 1990's) andthe negative outward in the Southern heliosphere (e.g during the 1960's and 1980's). Thus, the denotationof positive orientationfield isrepresented by A $>0$ epoch and a negative field is by the $\mathrm{A}<0$ epoch $[47,48]$. The transportation processes of cosmic ray particle in the solar wind plasma flow is the magnetic field of the heliosphere which are expressed in four distinct ways: An outward convection caused by radially-directed solar wind velocity; The magnetic field varies systematically over life scales were they can experience curvature and gradient drifts; Depending on the sign of the divergence of solar wind velocity were their energy can change; The spatial diffusions which causes scattering by random magnetic irregularities $[7 ; 49,50,51]$

\section{Solar Index}

Aninitial solar index of solar activity have been sunspot events. The events are extrinsic to the solar modulation of cosmic rays and other indices have been introduced with their merits discussed [52, 53, 54, 55].In this work, the solar index of solar activity used is the solar flare events which are measured in hours (hr) intervals for a year.

\section{Materials, Methods And Result Analysis}

The materials used in this work are from observatories of Mexico for cosmic ray data and Space Physics Interactive Data Resources (SPIDR) for solar flare data for the year 2004 respectively. These data were 
collected in hourly intervals for a duration of onemonth. Therefore, a month data have a maximum of $720 \mathrm{hrs}$. The process were repeated for 12- month's events in 2004. The data were arranged in four different sets in an excel spread sheets. In the spread sheets, the data were statistically analyzed and used in this paper. In each of the sets, a chosen cut off rigidity of 7000 counts for galactic cosmic rays(GCRs) and 100 counts for galactic solar flare(GSFs) were taken. The computations of GCRs and GSFs data were considered in each of the set. The sets were characterized as GCR (measured in counts) and GSF (measured in counts) against time (measured in hour). They are presented in a graphical form asshown in Fig.1-2. The first set covers the events from January, February and March of maximum of $0.744 \times 10^{2} \mathrm{hr}$ as shown in Fig. 1; the second set covers the events from April, May and June ofmaximum of $0.744 \times 10^{2} \mathrm{hr}$ as shown in Fig. 2; the third set covers the events from July, August and September of maximum ofmaximum of $0.768 \times 10^{2} \mathrm{hr}$ as shown in Fig.3; and the fourth set covers the events from October, November and December of maximum of $0.768 \times 10^{2} \mathrm{hr}$ as shown in Fig. 4 .

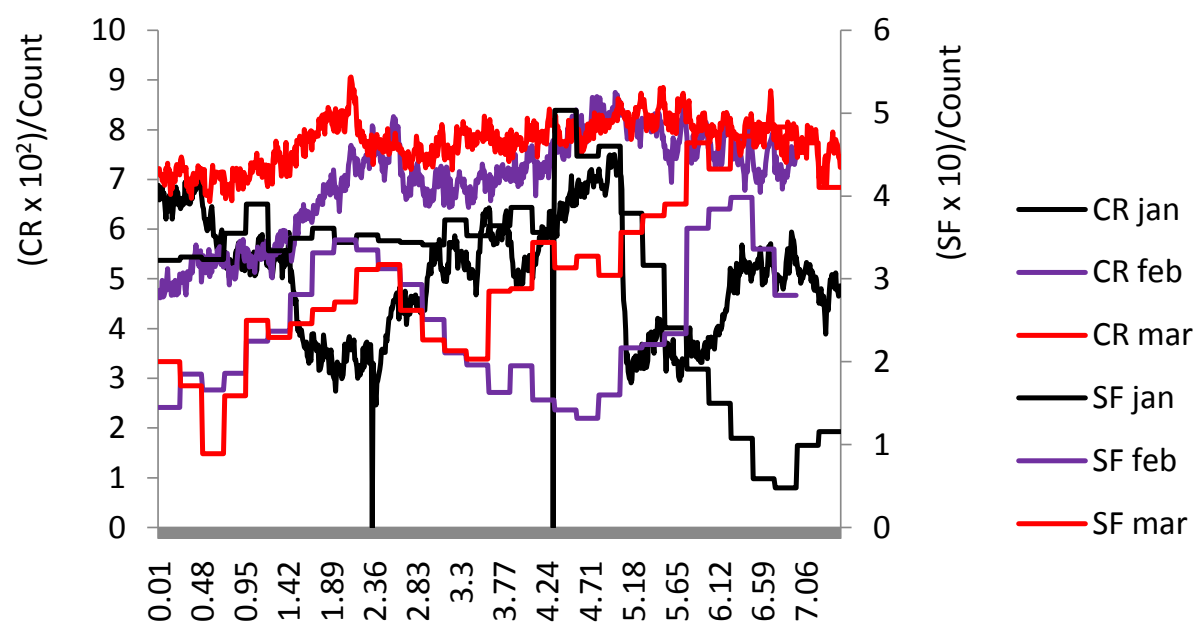

(Time $\left.\times 10^{2}\right)$ /hour

Fig.1. Variations of Galactic Cosmic Rays (GCRs) and Galactic SolarFlares (GSFs) against Time/hours. The legend colour keys for GCR (CR) and GSF (SF): the month of January is black; the month of February is blue andthe month of March red.

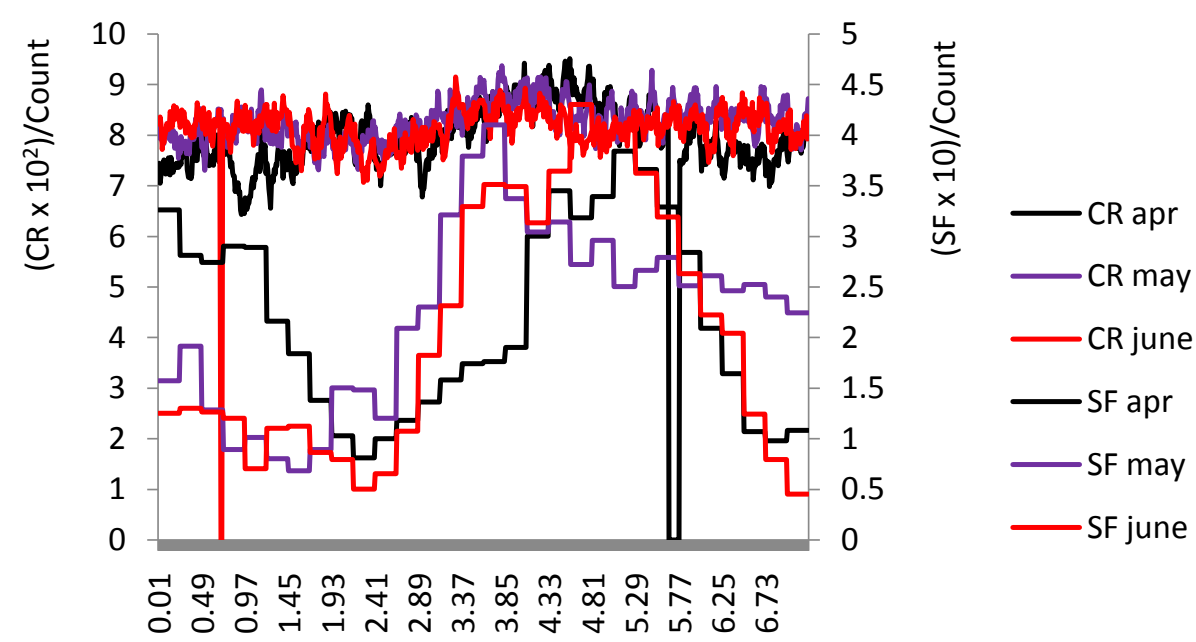

(Time $\left.\times 10^{2}\right) /$ hour

Fig.2. Variations of Galactic Cosmic Rays (GCRs) and Galactic Solar Flares (GSFs) against Time/hours. The legend colour keys for GCR (CR) and GSF (SF): the month ofApril is black; the month ofMay is blue andthe month ofJune is red. 


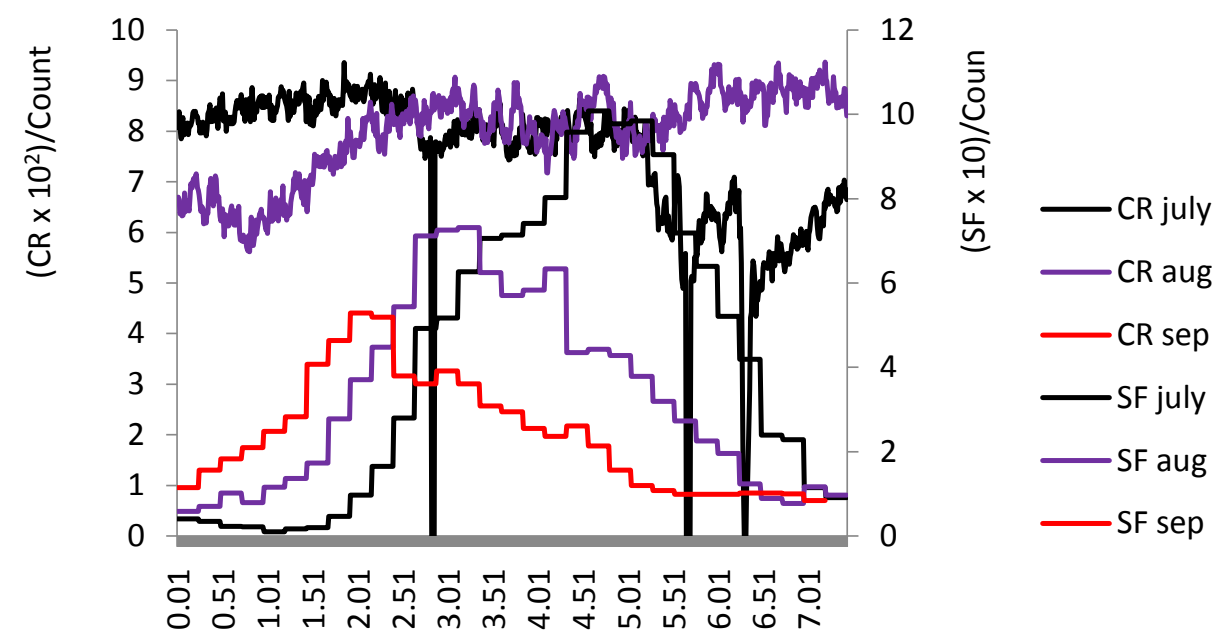

(Time $\left.\times 10^{2}\right)$ /hour

Fig.3 Variations of Galactic Cosmic Rays (GCRs) and Galactic Solar Flares (GSFs)against Time/hours. The legend colour keysfor GCR (CR) and GSF (SF): the month ofJuly is black; the month ofAugust is blue andthe month of September is red.

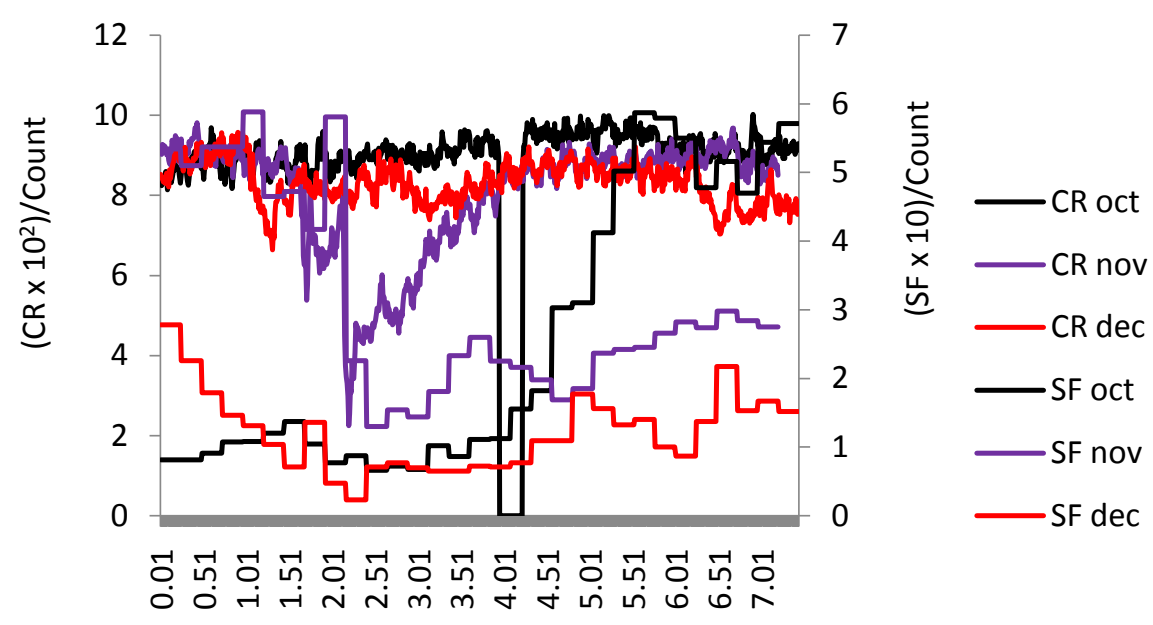

(Time $\left.\times 10^{2}\right)$ /hour

Fig.4 Variations of Galactic Cosmic Rays (GCRs) and Galactic Solar Flares (GSFs) against Time/hours. Thelegend colour keys for GCR (CR) and GSF (SF): the month ofOctober is black; the month ofNovemberis blue andthe month of December is red.

Furthermore, the correlation analysis were carried out in order to ascertain the level of relationship between GCRs and GSFsusing the excel program. The results of the correlation coefficient, $r$, ranges from 0.015 to -0.470 .

\section{Discussion And Conclusions}

The results of the statistical analysis of the two events (i.e. GCRs and GSFs) showed significantand clear characterization. The GCRs showed a continuous variation with time while the GSFs showed a small scale block variations with time in all the 8,808 hourly events in 2004.Thesevariations are in agreement with some authors [56, 57, 58, 59]. Some of the variation results show no GCRs detection: Fig.1 identified January (at $\sim 2.36$ and $\left.4.24 \times 10^{2} \mathrm{hrs}\right)$; Fig. 2 showed June $\left(\sim 0.64 \times 10^{2} \mathrm{hrs}\right)$ and April (ranging from $\left.\sim 5.59-5.86 \times 10^{2} \mathrm{hrs}\right)$; Fig.3 identified July $\left(\sim 2.83,5.65,6.31 \times 10^{2} \mathrm{hrs}\right)$ and Fig.4 showed October (ranging from $\left.3.95-4.24 \times 10^{2} \mathrm{hrs}\right)$. On the other side of the events, GSFs showed high significant detection in all the months.In comparing the variations between GCRs and GSFs:Fig.1 showed very high variations in GCRs between 0.01-0.48x 10² hrs and 2.83- 
5.18x $10^{2}$ hrs; but low between $1.42-2.83 \times 10^{2}$ hrs and 5.19-6.12x $10^{2}$ hrs. Thus, in February and March the variations in GCRs are almost high and flat. For GSFs, shows high variations in January between the ranges 4.24-5.18 $10^{2} \mathrm{hrs}$ and low between 6.12- 7.06 $10^{2} \mathrm{hrs}$. While in February and March, high variations ranging from 1.42 to $2.83 \times 10^{2} \mathrm{hrs}$ and low variations between $3.3-5.18 \times 10^{2} \mathrm{hrs}$;Fig. 2 showed that the GCRs variationsin April, May and June are almost flat and high, while the GSFs showed almost consistent high variations within the range 0.01-1.36x $10^{2} \mathrm{hrs}$ and 3.16-5.86x $10^{2} \mathrm{hrs}$, but low between $1.316-2.71 \mathrm{x} 10^{2} \mathrm{hrs}$ and $5.87-6.76 \mathrm{x}$ $10^{2} \mathrm{hrs}$;Fig.3 showed also flat and high variations of GCRs in July, August and September. Whereas GSFs show high variations between 1.42-2.36x $10^{2} \mathrm{hrs}$ in September and between 2.36-5.65x $10^{2} \mathrm{hrs}$ in the months of July and August and Fig.4 showed that GCRs are high and almost flat variation in October and December,while high variation occurred in November withinthe range $0.01-1.89 \times 10^{2} \mathrm{hrs}$ and $3.3-7.06 \times 10^{2} \mathrm{hrs}$, but low in the range 2.36-3.77 $\times 10^{2}$ hrs. Whereas, GSFsin the month of October are highwithin the range 5.18-7.06 $\times 10^{2}$ hrs and low between the range 1.01-4.27 $\times 10^{2} \mathrm{hrs}$.In addition, the correlation coefficients, $\mathrm{r}$ between GCRs and GSFs are anticorrelations which ranges from -0.015 to -0.450 . These anti-correlation coefficient range is in agreement with other authors [56, 57, 58,59].

In conclusions, the measurements of GCRs and GSFs on hourly events for the year, 2004. The interpretation of these measurements are being investigated. The graphical analysis showed that they are presents of GCRs and GSFs in the earth's atmosphere. The hourly variation of GCRs indicates a continuous arrival of events with variation in amplitude, except when arrivals are not recorded. This may be attributed to the instrumental rigidity $(\mathrm{GV})$. But, the hourly variation of GSFs is constantly continuous for some seconds, their amplitude also varies. Finally, the anti-correlation coefficient found in these results shows that the events originate from different source.

\section{Acknowledgement}

The author is grateful to the contributions of Mr. Okpaga Afamefuna M., Mr. Ukoji Nnanna U., Mr. Uda Callistus N., Mr. Nwuzor Ogochukwu C. and Mr. Eze Tochukwu, S.M. Finally, to my wife (Mrs. Lilian C. Umahi) and my children (Chidera, Sopuruchukwu, Kasiemobi, Ugochukwu and Chukwuebuka), for accommodating my long absence at home during the pursuit of this work.

\section{Reference}

[1]. Venkatesan, D., Badruddin, (1990). Space Sci. Rev. 52,121.

[2]. Cane, H.V., (1993), J. Geophys. Res. 98, 3509.

[3]. Potagieter, M.S, Buger, R.A., Ferreira, S.E.S (2001),Space Sci. Rev. 97, 295

[4]. Kudela, K., Storinic, M., Hofer, M. Y., Belov A. V., (2000),Space Sci. Rev. 93, 193

[5]. Kudela, K., (2009), Acta Physics Slovaca, 59, 537

[6]. Richardson, I.G., (2004), Space Sci. Rev. 111, 267.

[7]. Heber, B., (2011), Space Sci. Rev.doi: 10. 1007/s 11214-011-9784-x

[8]. Shikaze, Y.; Haino, S.; Abe, K.; Fuke, H.; Hams, T.; Kim, K. C.; Makida, Y.; Matsuda, S.; Mitchell, J. W.; Moiseev, A. A.; Nishimura, J.; Nozaki, M.; Orito, S.; Ormes, J. F.; Sanuki, T.; Sasaki, M.; Seo, E. S.; Streitmatter, R. E.; Suzuki, J.; Tanaka, K.; Yamagami, T.; Yamamoto, A.; Yoshida, T.; Yoshimura, K. (2007). Astropart. Phys., 28, 154

[9]. Umahi, A.E.(2016).Middle-East Journal of Scientific Research, 24 (5), 1788-1793. DOI: 10.5829/idosi.mejsr.2016.24.05.23456

[10]. Umahi, A.E.(2016).American-Eurasian J. Agric. \& Environ. Sci., 16 (5), 868-873, DOI: 10.5829/idosi.aejeas.2016.16.5.10440.

[11]. Bazilevskaya, G.A., I.G. Usoskin, E.O. Fluckiger, R.G. Harrison, L. Desorgher, et al., (2008), Space Sci. Rev., 137, 149-173.

[12]. Neher, H. V., (1971). J. Geophys. Res. 76, 1637-1651.

[13]. Lowder, W. M., Raft, P. D and Beck, H. L., (1972). Procs. National Symp. On Natural and Manmade Radiation in Space, NASA, 908-913.

[14]. Rosen, J. M., Hofmann, D. J., and Gringel, W., (1985). J. Geophys. Res., 90(D4), 5876-5884.

[15]. Ermakov, V.I., Bazilevskaya, G.A., Pokrevsky, P.E., Stozhkov, Yu.I. (1997). J. Geophys. Res., 102(D19), $23413-23419$.

[16]. Shea, M. A., Smart, D. F. (1996). Nuovo climento19c (6). 945-952.

[17]. Braun, I, Engle, J., Horandel J.R., Mike, J. (2005), $29^{\text {th }}$ int. cosmic ray conf., pune, 2, 135-138

[18]. Ajima,Y., Anraku,K.,Haga,T., Higashi,Y.,Honda,H. Imori,M.,Inaba, S., Kimura,N.,Kobayashi,M.,Makida, Y., Matsui,N., Matsumoto, H., Matsunaga,H.,Motoki, M., Nozaki,M.,Nishihara, Y., Nishimura,J., Orito,S.,Otoba, M., Saeki, T., Sanuki,T., Sasaki,M., Shimamura, K., Suzuki,J., Suzuki,K.,Takimi, N., Tanaka,K., Tsunoda, T., Ueda,I., Yajima,T.,Yamagami,T., Yamamoto,A., Yamaoka, H., Yoshida,T.,Yoshimura,K., (2000), Nucl. Instr. and Meth. A 443, 71

[19]. Umahi, A.E.(2016).Middle-East Journal of Scientific Research, 24 (5), 1794-1801.DOI:10.5829/idosi.mejsr.2016.24.05.23457.

[20]. Asaoka Y. et al. (1998), Nucl. Instr. and Meth. A 416, 236

[21]. Orito S. (1987), Proc. ASTROMAG Workshop, KEK Report KEK87-19, 111

[22]. Shikaze Y. et al. (2000), Nucl. Instr. and Meth. A 455, 596

[23]. Yamamoto A. et al. (1988), IEEE Trans. Magn. 24, 1421

[24]. Yamamoto A. et al. (1994), Adv. Space Res. 14(2), 75

[25]. Abe K. et al. (astro-ph/0304102) to be submitted to Phys. Lett. B

[26]. Papini P., Grimani C. and Stephens A. 1996, NUOVO CIMENT 19, 367

[27]. Forbush, S.E. (1954), J.Goephys. Res. 59, 525

[28]. Forbush, S.E., (1958), J. Geophys. Res. 63, 651.

[29]. Simpson, J.A., (1963), Proc. $8^{\text {th }}$ int. conf. cosmic rays, 2, 155.

[30]. Hatton, (1980). Solar Physics 66, 159-165.

[31]. Dorman, I.V., Dorman, L.I., (1967), J. Geophys. Res. 72, 1513.

[32]. Simpson, J.A., Wang, J.R., (1967), Astrophys. J. 149. L73 
[33]. Simpson and Wang, (1970). Astrophysical Journal, vol. 161, p.265.

[34]. Pomerantz, M.A., Duggal, S.P., (1974), Res. Geophys. Space phys. 12, 343.

[35]. Umahi, A. E. (2016).American-Eurasian J. Agric. \& Environ. Sci., 16 (5), 874-881, DOI: 10.5829/idosi.aejeas.2016.16.5.10441.

[36]. Burlaga, L.F., McDonald, F.B., Goldstien, M.L., Lazarus, A.J. (1985), J. Geophys. Res. 90, 12027.

[37]. Storinic, M., Borello-Filisetti, D., Mussinov, V., Parisi, M., Sykora, J., (1995), Solar phys. 157, 375

[38]. Ahluwalia, H.S., Wilson, M.D. (2001), J. Geophys. Res. 101, 4879.

[39]. Mavromichalaki, H., Belehaki, A., Rafios, X. (1998), Astron. Astrophys 330, 764

[40]. Cliver, E.W., Ling, A.G., (2001), Astrophys. J., 551, L189.

[41]. Kane, R.P., (2003), J. Geophys. Res., 108, 1379.

[42]. Sabbah, I., Rybansky, M., (2006) J. Geophys. Res. 111, A 01105.

[43]. Badruddin, S.M., Singh, Y.P., (2007). Astron. Astrophys. 466, 677.

[44]. Singh, M., Singh, Y. P., Badruddin (2008), J. Atmos. Solar-terr. Phys. 70, 169

[45]. Jokipic, J.R., Thomas, B.T. (1981),, 243, 1115.

[46]. Potgieter, M.S., Moraal, H (1985),Astrophys. J., 294, 425.

[47]. Duldig, M.L., (2001), Publ. Astron. Soc. Aust. 18, 12

[48]. Singh, Y.P., Badruddin, (2006), Solar phys. 234, 339.

[49]. McDonald, F.B., Nand Lal, Mcguire, R.E. (1993), J.Geophys. Res. 98, 1248., McDonald, F.B., Webber, W.R., Reames, D.V. (2010), Geophys. Res. Lett. 37, L18108, 1-5.

[50]. Potgieter, M.S., (1994), $23^{\text {rd }}$ int. cosmic ray conf., World Scientific, Singapore, 213.

[51]. McKibben, R.B., Connel, J.J., Lopate C., Simpson, J.A., Zhang, M., (1995), Space sci. Rev. $72,362$.

[52]. Sarabhari, V.A., Subramanian, G (1965), Proc. $10^{\text {th }}$ int. conf. cosmic rays $1,170$.

[53]. Eddy, J.A., Hansen, R.T. (1973). Proc. 13 ${ }^{\text {th }}$ int. conf. cosmic rays 2, 1184

[54]. Mendell, R.B., Korff, S.A., (1975), Proc. $14^{\text {th }}$ int. conf. cosmic rays, 4, 941.

[55]. Parker, G.D., Chasson, R.T., Hansen, R.T., Hansen, S.F., (1976), Solar phys. 48, 399. [56]. Usoskin, I, L., Desorgher, P. I.Y, Velinov, M. Storini, E., Flueckiger, R., Buetikofer and Kovalstov, G. A. (2009). Acta Geophys., 57, 88-101.

[56]. Velinov, P.I.Y., Asenovski, S., Kudela, K., Lastovicka, J., Mateev, L., Mishev, A. and Tonev, P., (2013). J. Space Weather Space Clim.3(A14), pp:1-17.

[57]. Umahi, A. E. (2016).World Applied Science Journal, 34 (3), 338-342, DOI:10.5829/idosi.wasj.2016.34.3.1565

[58]. Umahi, A. E. (2016).World Applied Science Journal, 34 (3), 312-317, DOI: 10.5829/idosi.wasj.2016.34.3.15660. 